\title{
Vaginal Lactobacilli for Mucosal Delivery of the Anti-HIV Microbicide, Cyanovirin-N (CV-N)
}

Qiang $\mathrm{Xu}^{* \neq 1}$, Laurel Lagenaur ${ }^{1}$, Xiaowen Liu ${ }^{1}$, David Simpson ${ }^{1}$, Kirsten Essenmacher ${ }^{1}$, Courtney Parker ${ }^{1}$, Chia-Hwa Chang ${ }^{1}$, Daniel Tsai ${ }^{1}$, Srinivas Rao ${ }^{2}$, Dean Hamer ${ }^{3}$, Thomas Parks ${ }^{1}$ and Peter Lee ${ }^{1}$

\author{
Address: ${ }^{1}$ Osel, Inc., 1800 Wyatt Dr., Ste. 14, Santa Clara, CA 95054 USA, ${ }^{2}$ Laboratory Animal Medicine, VRC, NIH, Bethesda, MD 20892 USA and \\ ${ }^{3}$ National Cancer Institute, NIH, Bethesda, MD 20892 USA \\ Email: Qiang Xu* - qxu@oselinc.com \\ * Corresponding author ‡Presenting author
}

from 2005 International Meeting of The Institute of Human Virology

Baltimore, USA, 29 August - 2 September 2005

Published: 8 December 2005

Retrovirology 2005, 2(SuppI I):S87 doi:I 0.1 I86/1742-4690-2-SI-S87

\section{Background}

Women are particularly at risk of HIV infection and there is an urgent need for female-controlled approaches to block the heterosexual transmission of HIV.

\section{Material and Methods}

Our work is aimed at the development of a simple, costeffective, female-controlled preventative against heterosexual transmission of HIV in women, based on our previous proof-of-concept study employing a natural component of the vaginal microflora, as a delivery vehicle for the anti-HIV protein (PNAS, 2003, 100:1167211677).

\section{Results}

A human vaginal isolate of Lactobacillus jensenii was engineered, by stable integration of an optimized expression cassette into the bacterial genome, to secrete high levels of the highly potent HIV inhibitor, CV-N. The L. jenseniiexpressed CV-N dramatically decreases infectivity of CCR5-tropic $\mathrm{HIV}_{\mathrm{BaL}}$ and CXCR4-tropic $\mathrm{HIV}_{\mathrm{IIIB}}$ in vitro. We further demonstrate that this strain is genetically stable and can transiently colonize animal vaginal mucosa, while retaining important characteristics of the native bacterial phenotype.

\section{Conclusion}

This live microbicide represents a novel approach in the development of an inexpensive and stable protein-based microbicide to curtail the HIV/AIDS pandemic worldwide. 